\title{
Comparative study of groin hernia repair
}

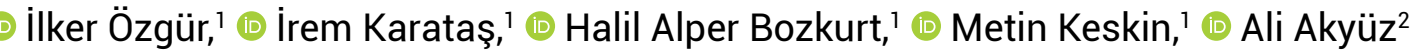 \\ 'Department of General Surgery, İstanbul Faculty of Medicine, İstanbul University, İstanbul, Turkey \\ 2Department of General Surgery, Acıbadem International Hospital, İstanbul, Turkey
}

\begin{abstract}
Introduction: The aim of the study is to compare results of Lichtenstein repair, laparoscopic totally extraperitoneal (TEP), and transabdominal preperitoneal (TAPP) repair of groin hernias.

Materials and Methods: We retrospectively extracted medical records of 127 consecutive male patients operated on for inguinal hernia between June 2014 and June 2019. Patients were grouped according to preferred surgery; Lichtenstein (Group 1), TAPP (Group 2), and TEP (Group 3) repair groups. Demographic data, body mass index (BMI), American society of anesthesiologists score, surgery time, post-operative hospital stay, pain score, complications, and recurrence rates were compared.
\end{abstract}

Results: Mean age was 53.6 (17-86) years. Mean BMI was $24.8 \mathrm{~kg} / \mathrm{m}^{2}$. Mean surgery time was $62.0 \pm 24.2$ (30-150), $71.1 \pm 26.9(40-135)$, and $76.5 \pm 20.9$ (35-1 40) min in groups, respectively. Mean hospital stay was $1.3(1-3), 1.2(1-4)$, and $1.3(1-2)$ days. Post-operative pain was less in laparoscopic group, especially in TEP repair group ( $1.0 \pm 0.9$ [0-4], $0.4 \pm 0.8$ [0-3], $0.2 \pm 0.6$ [0-3]; 1 vs. 2 p: $0.005 / 2$ vs. 3 p: $0.000 / 1$ vs. 3 p: $0.001)$. No major complication was observed and two recurrent hernias were detected after mean 953-day follow-up period.

Conclusion: Both open and laparoscopic hernia repair can be performed safely, with a low rate of complications. Post-operative pain is reduced in the laparoscopic group.

Keywords: Inguinal hernia, Lichtenstein repair, Transabdominal preperitoneal, Totally extraperitoneal

\section{Introduction}

Annual hernia repair is reported more than 20 million cases worldwide. ${ }^{[1]}$ Several methods were described after the first operation held by Bassini in 1887 but still, no consensus exists on the ideal or best surgery type. Mesh repair methods accumulatively have increased to be preferred by surgeons due to decreased rates of recurrence reported by Lichtenstein. ${ }^{[2]}$ Laparoscopic repair became the most preferred surgery type due to low rates of post-operative pain, better cosmesis, and early return to work. ${ }^{[3]}$ Howev- er, cost and long learning curve debate the utilization of laparoscopy. However, long-term cost and benefit seem to be similar to open techniques when the quality of life or total community cost is considered. ${ }^{[1]}$

\section{Materials and Methods}

We retrospectively analyzed 127 male patients operated on for an inguinal hernia between June 2014 and June 2019. All surgeries were on an elective basis. Patients' comorbidities or desire for open surgery enrolled them in the Lichtenstein 
repair (LR) group (Group 1). Patients' desire and agreement for laparoscopic surgery enrolled them in the transabdominal preperitoneal (TAPP) repair group (Group 2) or totally extraperitoneal (TEP) repair group (Group 3) depending on surgeons' choice. Two surgeons performed the operations, and both performed the three procedures. Hernia defect was covered with polypropylene mesh. Paracetamol was preferred for post-operative pain management in a dose of 3 times $500 \mathrm{mg}$ intravenously during hospitalization and the same dose orally for a week after discharge from the hospital. No other pain killer was administered. Demographic data, body mass index (BMI), American society of anesthesiologists (ASA) score, surgery time, and post-operative complications were compared.

\section{Ethical Aspects}

The study protocol was approved by the Istanbul University Ethics Committee with decision number 31/18.12.2020. The study was conducted in accordance with the principles of the Declaration of Helsinki. All patients gave informed consent.

\section{Statistical Analysis}

Statistical analysis was extracted from the excel Microsoft database. The continuous variables were expressed as mean and standard deviation and categorical variables as frequencies and percentages. Categorical values were calculated with Chi-square and Fisher's exact test. Three group comparisons were done with ANOVA. Statistical significance of $P$ values level was assumed as $<0.05$. Statistical analyses were performed with IBM ${ }^{\circledR}$ SPSSv22 software (IBM Corporation, Armonk, NY, USA).

\section{Results}

The mean age of all patients was $53.6 \pm 15.1(17-86)$ years and there was no statistical difference between groups $(\mathrm{p}=0.087)$. BMI $(\mathrm{p}=0.744)$ and ASA scores $(\mathrm{p}=0.076)$ were similar in the three groups. The right hernia was more common than the left-sided one in all groups. The bilateral hernia was significantly higher in the TEP group than in the other two groups (Table 1).

The mean duration of surgery was shorter in the LR group, 62 24 (30-150), and had statistical significance

Table 1. Patients' characteristics

\begin{tabular}{|c|c|c|c|c|}
\hline & Group 1 & Group 2 & Group 3 & Total \\
\hline Patients (n, \%) & $46(36.2)$ & $22(17.3)$ & $59(46.5)$ & 127 \\
\hline \multirow[t]{2}{*}{ Age, years } & $54.1 \pm 17.5$ & $52.3 \pm 16.1$ & $53.7 \pm 12.8$ & $53.6 \pm 15.1$ \\
\hline & $(21-85)$ & $(17-80)$ & $(24-86)$ & $(17-86)$ \\
\hline \multicolumn{5}{|l|}{ ASA score (n) } \\
\hline I & 19 & 13 & 37 & 69 \\
\hline II & 24 & 7 & 21 & 52 \\
\hline III & 3 & 2 & 1 & 6 \\
\hline \multirow[t]{2}{*}{$\mathrm{BMI}, \mathrm{kg} / \mathrm{m}^{2}$} & $24.8 \pm 3.0$ & $24.4 \pm 3.9$ & $25.0 \pm 3.0$ & $24.8 \pm 3.1$ \\
\hline & $(18-32)$ & $(18-37)$ & $(18-33)$ & $(18-37)$ \\
\hline Redo surgery & 2 & 1 & 4 & 7 \\
\hline Bilateral (n) & 4 & 7 & 41 & 52 \\
\hline Right (n) & 26 & 12 & 13 & 51 \\
\hline Left (n) & 16 & 3 & 5 & 24 \\
\hline Total sides (n) & 50 & 29 & 100 & 179 \\
\hline Recurrence (n) & 0 & 1 & 1 & 2 \\
\hline Direct hernia(n) & $17 / 20 *$ & $2 / 7 *$ & $5 / 35 *$ & $24 / 62 *$ \\
\hline Indirect hernia(n) & $22 / 27 *$ & $12 / 21 *$ & 10/49* & 49/97* \\
\hline Pantaloon hernia (n) & $3 / 3 *$ & $1 / 1 *$ & $2 / 15^{*}$ & $6 / 19 *$ \\
\hline Femoral-obturator (n) & $1-0 / 1 *-0 *$ & $0-0 / 0 *-0 *$ & $1-0 / 1 *-1 * *$ & $1-0 / 1 *-1 * *$ \\
\hline
\end{tabular}


when compared to the TEP repair group. When surgery time of only single-sided or only bilateral-sided surgeries was compared between surgical procedures there was no statistical difference between groups (Table 2). Hospitalization time was similar in the three groups. Post-operative pain was least in the TEP repair group and less in the TAPP group when compared to the LR group.

Twelve complications were observed in the study group during the mean follow-up of $1251 \pm 483$ (292-2150), 995 \pm 384 (350-1919), and 953 484 (243-1937) days, respectively. The complication rate was similar between groups, 3, 3, and 4 ( $\mathrm{p}=0.191$ ), respectively. Scrotal edema, hematoma, and seroma were observed in the LR group. In TAPP repair group scrotal edema and seroma developed in two patients. One patient suffered from scrotal edema and another one from seroma in the TEP repair group. Chronic pain was observed in one patient in the TEP group and it ceased with the nonsteroidal anti-inflammatory drug over 4 months. One recurrence was observed in the LR group and TEP repair group each.

\section{Discussion}

Inguinal hernia repair has changed from primary tissue repair to tension-free repair in approximately 50 years. More recently patch repair was added. The primary goal of this evolution was to achieve fewer relapses. Today, surgeons are not just on fewer relapses. At the same time, they try to define the surgical method, which is less expensive, has a short hospital stay, painless in the postoperative period, and has good cosmetic results and few complications. The seek for a "gold standard" has been studied for more than 100 years and still, there is no consensus. We retrospectively reviewed files of 127 patients operated for inguinal hernia and followed between August 2014 and August 2019 in our clinic. Patients and surgeries performed were compared.

Hamza et al. ${ }^{[4]}$ and Dedemadi et al. ${ }^{[5]}$ reported longer surgery time with laparoscopy, while Wang et al. ${ }^{[6]}$ did not report a statistical difference in the duration of surgery. Our study demonstrated similar operative times and there

\section{Table 2. Intergroup comparison of surgery time, hospital stay, VAS score, and complications}

\begin{tabular}{|c|c|c|c|c|}
\hline & Group 1 & Group 2 & Group 3 & $\mathbf{p}$ \\
\hline \multirow[t]{3}{*}{ Surgery time, $\min$} & $62.0 \pm 24.2$ & $71.1 \pm 26.9$ & $76.5 \pm 20.9$ & 1 vs 20.289 \\
\hline & $(30-150)$ & $(40-135)$ & $(35-140)$ & 2 vs 30.593 \\
\hline & & & & 1 vs 30.04 \\
\hline Single sided only & $58.2 \pm 19.4$ & $60.4 \pm 17.01$ & $68.6 \pm 23.5$ & 1 vs 20.986 \\
\hline \multirow[t]{2}{*}{ surgery time, $\min$} & $(30-120)$ & $(40-95)$ & $(35-130)$ & 2 vs 30.308 \\
\hline & & & & 1 vs 30.686 \\
\hline Bilateral sided only & $102.5 \pm 35.7$ & $94.2 \pm 30.7$ & $80.4 \pm 18.8$ & 1 vs 20.924 \\
\hline \multirow[t]{2}{*}{ surgery time, $\min$} & $(75-150)$ & $(50-135)$ & $(40-140)$ & 2 vs 30.378 \\
\hline & & & & 1 vs 30.193 \\
\hline \multirow[t]{3}{*}{ Hospital stay, days } & $1.3(1-3)$ & $1.2(1-4)$ & $1.3(1-2)$ & 1 vs 20.487 \\
\hline & & & & 2 vs 30.771 \\
\hline & & & & 1 vs 30.799 \\
\hline \multicolumn{5}{|l|}{ Pain Score (VAS) } \\
\hline $1^{\text {st }} \mathrm{hr}$ & $2.6 \pm 2.2(0-8)$ & $3.9 \pm 1.8(0-8)$ & $1.0 \pm 0.9(0-4)$ & 1 vs $20.09 / 2$ vs $30.000 / 1$ vs 30.000 \\
\hline $6^{\text {st }} \mathrm{hr}$ & $1.6 \pm 1.7(0-5)$ & $3.1 \pm 1.6(0-6)$ & $0.4 \pm 0.8(0-3)$ & 1 vs $20.000 / 2$ vs $30.000 / 1$ vs 30.000 \\
\hline $1^{\text {st }}$ day & $0.9 \pm 1.1(0-4)$ & $1.7 \pm 0.9(0-4)$ & $0.2 \pm 0.6(0-3)$ & 1 vs $20.005 / 2$ vs $30.000 / 1$ vs 30.001 \\
\hline Complications (n, \%) & $3(6.5)$ & $3(13.6)$ & $4(6.7)$ & 0.191 \\
\hline Scrtotal edema & $1(2.1)$ & $1(4.5)$ & $1(1.7)$ & \\
\hline Seroma & $1(2.1)$ & $1(4.5)$ & $1(1.7)$ & \\
\hline Hematoma & $1(2.1)$ & - & - & \\
\hline Recurrence & - & $1(4.5)$ & $1(1.7)$ & \\
\hline Chronic pain & - & - & $1(1.7)$ & \\
\hline
\end{tabular}


was no statistical difference in the three groups. When unilateral or bilateral hernia was considered separately, also there was no statistical difference except the bilateral hernia group. TAPP procedure lasted longer when compared to the TEP procedure. This prolongation of operative time may arise due to the preparation of preperitoneal space and the need for peritoneal closure after patch placement. Recent guidelines based on meta-analyses and randomized control trials showed no difference in terms of operative time. The median operation time was $57 \mathrm{~min}$ (34.5-104.5) for TAPP and $62.3 \mathrm{~min}$ (32.5-110) for TEP techniques. ${ }^{[7]}$

There are several different reports on hospital stay after laparoscopic surgery for inguinal hernia repair. International Endohernia Society reported TAPP with a longer hospital stay ${ }^{[8]}$ Similarly, a study from Switzerland held by Gass et al. reported statistically longer hospitalization (2.9 vs. 2.3 days; $p=0.002$ ) of TAPP group patients. ${ }^{[9]}$ In contrast, Wittenbecher and friends figured out shorter hospitalization in TAPP patients. ${ }^{[10]}$ Furthermore, some studies do not describe superiority in terms of hospital stay. ${ }^{[11]}$ In our study, there was no statistical difference between the three groups.

Early and late complications are reported in different ranges defining the superiority of both laparoscopic techniques on each other. ${ }^{[4-7,9,11-13]}$ Although the complication rate is reported, as high as $50 \%$ in both laparoscopic surgery types overall reported median is nearly $12-13 \%$. ${ }^{[1]}$ We observed one scrotal edema and one seroma formation in each group. Hematoma developed in one patient and vanished with no intervention in the LR group. Pain lasting longer than 3 months at the upper thigh dermatome was observed in one patient and a nonsteroidal anti-inflammatory drug was prescribed. Later on, the pain decreased and the patient was free of pain after $4^{\text {th }}$ month.

A large German study conducted by Köckerling et al. ${ }^{[7]}$ reported vascular, bladder, and bowel injury that happened more commonly in the TAPP group versus TEP $(0.71 \%$ vs. $=0.63 ; \mathrm{p}=0.570)$. The same study reported post-site hernias which were more common after TAPP $(0.4 \%)$ than TEP $(0.026 \%)$. No access related complications or access point hernia was observed in our study group.

Different recurrence rates with decreasing ratios over time probably due to the learning curve are reported in the literature. ${ }^{[1]}$ The expected recurrence rate is below $2 \%$. In our study, one patient in the TEP study group and one in the LR group developed early recurrence. No late recurrence was observed.

Several studies compared early and chronic post-operative pain and reported no statistical difference between open and laparoscopic or inter laparoscopic groups. ${ }^{[6,13-15]}$ Our study group developed significantly less pain after TEP and LR groups.

Our study has some limitations. The retrospective design of the study may contribute to some bias due to gathered memory data. Second is the presence of a large number of patients with bilateral hernias in the TEP group. Such a large number of patients developed due to ultrasound claims of the insurance companies before the surgery. Confirmation of opposite site hernia and willingness of the patient of opposite site surgery contributed to the proportion of this high value of bilateral hernia.

Despite all the limitations of most comparative studies and conducted meta-analyses and systemic reviews concluded that there is no superior technique to recommend over each other due to insufficient evidence. ${ }^{[1]}$ Each technique has rare and different complication risks. Surgeon experience and ease have an important role in technique preference for the individualized patient. Each technique may be performed preferentially. ${ }^{[16,17]}$

Minimal invasive era mandated laparoscopic surgery for hernia repair. It is clear that laparoscopic surgery has bare advantages in terms of post-operative pain and recovery speed when compared to open surgery. In case of the inadequate experience of the surgeon or low volume center, open surgery should be preferred for safeness and shorter operation time. Sufficient experience in each technique ends with comparable outcomes. The surgical method for inguinal hernia repair should be individualized based on the experience of the surgeon, patient preferences, and case characteristics.

\section{Disclosures}

Ethichs Committee Approval: The study protocol was approved by the Istanbul University Ethics Committee with decision number 31/18.12.2020. The study was conducted in accordance with the principles of the Declaration of Helsinki. All patients gave informed consent.

Peer-review: Externally peer-reviewed.

Conflict of Interest: None declared. 
Authorship Contributions: Concept - İ.Ö., M.K., A.A.; Design - İ.Ö., M.K., A.A.; Supervision - İ.Ö., M.K., A.A.; Materials - İ.Ö., M.K., A.A.; Data collection and/or processing - İ.Ö., İ.K., H.A.B.; Analysis and/ or interpretation - İ.Ö., M.K., A.A.; Literature search - İ.Ö., İ.K., H.A.B.; Writing - İ.Ö., İ.K., H.A.B., M.K., A.A.; Critical review İ.Ö., M.K., A.A.

\section{References}

1. HerniaSurge Group. International guidelines for groin hernia management. Hernia 2018;22:1-65. [CrossRef]

2. Lichtenstein IL. Herniorrhaphy. A personal experience with 6,321 cases. Am J Surg 1987;153:553-9. [CrossRef]

3. Heikkinen TJ, Haukipuro K, Koivukangas P, Hulkko A. A prospective randomized outcome and cost comparison of totally extraperitoneal endoscopic hernioplasty versus Lichtenstein hernia operation among employed patients. Surg Laparosc Endosc 1998;8:338-44. [CrossRef]

4. Hamza Y, Gabr E, Hammadi H, Khalil R. Four-arm randomized trial comparing laparoscopic and open hernia repairs. Int J Surg 2010;8:25-8. [CrossRef]

5. Dedemadi G, Sgourakis G, Karaliotas C, Christofides T, Kouraklis G, Karaliotas C. Comparison of laparoscopic and open tension-free repair of recurrent inguinal hernias: A prospective randomized study. Surg Endosc 2006;20:1099-104.

6. Wang WJ, Chen JZ, Fang Q, Li JF, Jin PF, Li ZT. Comparison of the effects of laparoscopic hernia repair and Lichtenstein tension-free hernia repair. J Laparoendosc Adv Surg Tech A 2013;23:301-5. [CrossRef]

7. Köckerling F, Bittner R, Jacob DA, Seidelmann L, Keller T, Adolf $D$, et al. TEP versus TAPP. Comparison of the perioperative outcome in 17,587 patients with a primary unilateral inguinal hernia. Surg Endosc 2015;29:3750-60. [CrossRef]

8. Bittner R, Montgomery MA, Arregui E, Bansal V, Bingener J, Bisgaard T, et al. Update of guidelines on laparoscopic (TAPP) and endoscopic (TEP) treatment of inguinal hernia (International Endohernia Society). Surg Endosc 2015;29:289-321.

9. Gass M, Banz VM, Rosella L, Adamina M, Candinas D, Güller
U. TAPP or TEP? Population-based analysis of prospective data on 4,552 patients undergoing endoscopic inguinal hernia repair. World J Surg 2012;36:2782-6. [CrossRef]

10. Wittenbecher F, Scheller-Kreinsen D, Röttger J, Busse R. Comparison of hospital costs and length of stay associated with open-mesh, totally extraperitoneal inguinal hernia repair, and transabdominal preperitoneal inguinal hernia repair: An analysis of observational data using propensity score matching. Surg Endosc 2013;27:1326-33. [CrossRef]

11. Bansal VK, Krishna A, Manek P, Kumar S, Prajapati O, Subramaniam $\mathrm{R}$, et al. A prospective randomized comparison of testicular functions, sexual functions and quality of life following laparoscopic totally extra-peritoneal (TEP) and tran$\mathrm{s}$-abdominal pre-peritoneal (TAPP) inguinal hernia repairs. Surg Endosc 2017;31:1478-86. [CrossRef]

12. Pokorny $H$, Klingler A, Schmid T, Fortelny R, Hollinsky $C$, Kawji $\mathrm{R}$, et al. Recurrence and complications after laparoscopic versus open inguinal hernia repair: Results of a prospective randomized multicenter trial. Hernia 2008;12:385-9. [CrossRef]

13. Krishna A, Misra MC, Bansal VK, Kumar S, Rajeshwari S, Chabra A. Laparoscopic inguinal hernia repair: Transabdominal preperitoneal (TAPP) versus totally extraperitoneal (TEP) approach: A prospective randomized controlled trial. Surg Endosc 2012;26:639-49. [CrossRef]

14. Zanghì A, Di Vita M, Lo Menzo E, Castorina S, Cavallaro AS, Piccolo $\mathrm{G}$, et al. Multicentric evaluation by verbal rate scale and EuroQoL-5D of early and late post-operative pain after TAPP and TEP procedures with mechanical fixation for bilateral inguinal hernias. Ann Ital Chir 2011;82:437-42.

15. Tolver MA, Rosenberg J, Bisgaard T. Early pain after laparoscopic inguinal hernia repair. A qualitative systematic review. Acta Anaesthesiol Scand 2012;56:549-57. [CrossRef]

16. Miserez M, Peeters E, Aufenacker T, Bouillot JL, Campanelli $\mathrm{G}$, Conze J, et al. Update with level 1 studies of the European Hernia Society guidelines on the treatment of inguinal hernia in adult patients. Hernia 2014;18:151-63. [CrossRef]

17. Bobrzynski A, Budzynski A, Biesiada Z, Kowalczyk M, Lubikowski J, Sienko J. Experience-the key factor in successful laparoscopic total extraperitoneal and transabdominal preperitoneal hernia repair. Hernia 2001;5:80-3. [CrossRef] 